\title{
Management of Compressed Air to Reduce Energy Consumption Using Intelligent Systems
}

\author{
Mohamad Thabet ${ }^{1}$, David Sanders ${ }^{1}$, Malik Haddad ${ }^{1}$, Nils Bausch ${ }^{1}$, \\ Giles Tewkesbury ${ }^{1}$, Victor Becarra ${ }^{1}$, Tom Barker ${ }^{2}$ and Jake Piner ${ }^{3}$ \\ 1 University of Portsmouth, Portsmouth, PO1 2UP, UK \\ 2 Gems Sensors, Lennox Road, Basingstoke, RG22 4AW, UK \\ 3 Watton Farm, Southampton SO32 3HA, UK
}

\begin{abstract}
This research investigated the use of intelligent systems for reducing energy consumption in compressed air systems. An initial literature review has been completed and mathematical models that describe typical compressed air components (compressor, tank, piping network, etc.) were created. The investigations suggested that energy used or wasted in connection with compressed air was a valuable research area to attempt to save energy. The research progressed to investigating ways of minimising energy use for air compressors based on real-time conditions (including anticipated future requirements), using intelligent systems to monitor and make decisions.
\end{abstract}

Keywords: Systems, Intelligent, Efficiency, Air, Energy.

\section{$1 \quad$ Introduction}

The research described in this paper aims to create innovative intelligent methods to reduce energy consumption in a compressed air system (CAS). The current goal is to combine ambient sensing information with artificial intelligence (AI) and knowledge management (KM) in real time. This is expected to increase the efficiency of energy intensive manufacture. Comprehensive information about performance will be provided from ambient sensors (data sources), which will be used by an AI systems to make automated decisions. Information will be processed by a KM system and human operators will be provided with advice on how to maintain productivity and reduce energy usage. The aims and objectives are summarised as follows:

1. Use AI and ambient sensing to evaluate and monitor performance

2. Use KM to decrease energy use.

3. Construct a collaborative interface for the human operators.

Air compressors alone account for over $10 \%$ of UK industrial energy use (>15,000 GWh) [1-2]. Industry is faced with high energy costs and needs to reduce both the financial and environmental impact of using energy. The scientific community can help by introducing new technologies and paradigms to create a step improvement in the energy efficiency of manufacturing industries.

CAS are often the most expensive and inefficient industrial systems. For every 10 units of energy supplied during the creation of compressed air, fewer than one unit is actually turned into useful compressed air [1,3]. This research will minimise energy use for air compressors based on real-time manufacturing conditions.

Over a 10 year life cycle, energy accounts for $75 \%$ of the cost of running a CAS [4]. Many of these systems could operate more efficiently, leading to energy savings and more reliable supply of compressed air [4]. Improving energy performance of these systems has been of interest to many researchers, with some studies claiming that savings of $20-50 \%$ may be achievable [5].

This paper includes a general literature review on research concerned with improving energy efficiency of CAS using intelligent energy management techniques. Moreover, mathematical models to evaluate energy performance of CAS were developed. Our research is now concentrating on the analysis and automatic detection of malfunctions (such as leaks) that negatively impact energy performance. Ongoing and future research at the University of Portsmouth will combine real time ambient sensing with artificial intelligence (AI) and knowledge management (KM) to automatically improve efficiency, especially in energy intensive manufacturing where compressed air is widely used. Ambient data will provide information on performance and AI will make sense of that data and automatically act. KM will facilitate the processing of information to advise human operators on actions to reduce energy use and maintain productivity. Some decisions might be made automatically, for example to increase pressure or shut down a compressor if a leak is detected.

Characteristics of inefficiencies in CAS are being considered and the new work will address automatic detection and decision making. Compressed air leaks, heat waste and overall inefficiencies within the process are some of the major causes of energy waste in CAS. By creating new methods for automatic detection and performance optimisation, a new framework for energy management of compressed air systems may be realised.

Section two of this paper reviews similar research in the area of intelligent energy management of CAS. Section three describes the gaps identified from the literature review. Section four begins to address the gaps by creating MATLAB mathematical models for CAS components. Sections five and six are the discussion and conclusion respectively. 


\section{Review of Similar Research}

Traditionally research in energy efficiency of CAS has focused on methods to reduce energy waste, approaches to model and simulate CAS and the development of tools and methods to identify air leakage.

During the past three decades, energy management systems were gradually being introduced into companies and facilities, mainly to decrease high economic and environmental costs in energy exhaustive processes [6]. These systems control energy consumption by evaluating performance, identifying malfunctions or energy efficiency opportunities and recommending curative actions.

Because complex systems such as CAS have an inherent variability in their energy consumption, are influenced by many factors and require regular maintenance, controlling their energy performance for long periods of time can be challenging. These challenges, in addition to other factors, incentivised an interest in self-learning energy management and automation technologies [6].

Santolamazza investigated the energy and performance optimization of CAS with machine learning [7-9]. In [7], a methodology to monitor and control energy performance in industrial plants was presented. The methodology applied a sequence of steps that supported the identification of changes in energy consumption patterns or decline in energy performance due to faults. In [8], Artificial Neural Networks (ANNs) to monitor energy performance of CAS and detect failure were implemented. Failures are typically preceded with irregularities in energy usage. It was concluded that ANNs were capable of characterizing system behavior, allowing the association of irregularities in performance with their causes through a control chart. In [9], Santolamazza studied three methods to monitor and control energy consumption in CAS, two machine learning approaches, ANNs and support vector machines (SVM), and a classical statistical approach. The results demonstrated that machine learning techniques allowed detection of anomalies, failure analysis and prescriptive maintenance, whereas statistical methods were mainly effective in identifying major anomalies.

Santolamazza's concluded that data collected from CAS and operating environments, could help in the detection of abnormalities (faults or energy inefficiencies) and in the suggestion of suitable counter measures. However, the association of these irregularities with their probable causes, and the generation of a troubleshooting procedure, were not examined comprehensively. Moreover, the suggested methodology and algorithm, were not validated experimentally, and therefore their effectiveness in real time remained subject to confirmation through positive experimentation.

Energy management systems may be combined with sensors and information systems that assist with collecting and analyzing data. Boehm and Franke investigated the concept of cyber physical CAS (CPCAS) [10]. CPCAS are industrial CAS armed with automation equipment and AI. These systems obtain basic operating parameters (such as pressure, volume, temperature, etc.) and assist in achieving more efficient operation. Further research into technical characteristics and specifications of CPCAS components was required.

Other researchers investigated the use of machine learning in compressor design and monitoring. Ghorbanian investigated the construction of compressor design performance maps using ANNs instead of costly and time consuming experimental methods [11]. ANN approach results agreed with the experimental data, while reducing development time and cost at initial design stages. Study in [11] investigated design and monitoring of compressor and the approach used may be applied to other CAS components.

The control and monitoring of CAS using data and intelligent technologies is an emerging field [6]. A major gap in research in this specific field included the creation of algorithms capable of detecting abnormalities in performance while associating those abnormalities with appropriate causes and developing a troubleshooting procedure. Another gap in research was the development of CPCAS characteristics and specifications of CPCAS. CPCAS may reduce energy usage however, they required more research into system components and functionalities.

\section{Gaps in Literature}

Six gaps in research were identified:

1- Ideas about the measures to reduce energy consumption are well developed theoretically in the literature but many are not realized, due to different barriers [12]. One of these barriers, is the high cost of gathering information and deciding on the most cost effective and applicable measures. Traditionally manufacturers look for energy saving opportunities through expensive and time consuming energy audits. A methodology to support decision making regarding suitable measures to save energy in CAS may result in a useful and innovative tool for enhanced energy efficiency.

2- Evaluation of CAS performance may be achieved through modelling and simulating, however most models in the literature model either the supply or demand side separately. Coupling compressed air supply and demand in a single model may be investigated in future research. Moreover, such a model could integrate and combine other energy consuming equipment (heating, cooling, lighting, etc.) with CAS.

3- Investigations into intelligent CAS energy management technologies has been gaining momentum. Machine learning techniques proved to be a suitable tool for CAS performance optimization by detecting anomalies in their performance but that was not investigated thoroughly. An energy management system that detects abnormalities in energy performance, associates the abnormalities with suitable causes and sets up a troubleshooting procedure has not yet been established.

4- Intelligent energy management technologies for CAS anomaly detection and prescriptive maintenance using machine learning and manufacturing data has not been validated experimentally. To properly test the effectiveness of such energy management system, future work needs to experimentally validate the theoretical work. 
5- The concept of cyber physical CAS (CPCAS) was introduced in [10]. CPCAS are equipped with additional components, such as sensors, actuators and processors for autonomous control. In addition, they are capable of exchanging information with energy management systems, and therefore may play a role in the intelligent energy management of CAS. However, the concept of CPCAS was not well developed and there is a gap in research regarding technical and functional characteristics of the components.

6- Compressed air leaks are a major source of energy waste in CAS and therefore their detection and treatment is an essential step in reducing energy waste. Compressed air leak detection technologies face many challenges including: incapability of operating during production, inaccuracy in sensors and noise from operating environments. Moreover, technologies such as ultrasound and acoustic leakage detection, fail to effectively identify leaks from small size openings [13]. Future research could further develop these technologies to improve accuracy, ease of use and range of applicability.

\section{$4 \quad$ Modelling CAS Equipment}

Compressed air systems consist of a supply and demand side. The supply side converts inlet air into compressed air, and typically includes equipment such as compressors, dryers, filters and coolers. The demand side delivers required compressed air to end users and normally includes piping networks, controllers and end use equipment [14].

A variety of sub-components make up a CAS, and numerous system configurations are possible. In this section, a basic CAS consisting of a compressor, cooler, filter, storage tank and distribution network is considered. A schematic diagram of such a system is shown in Fig.1.

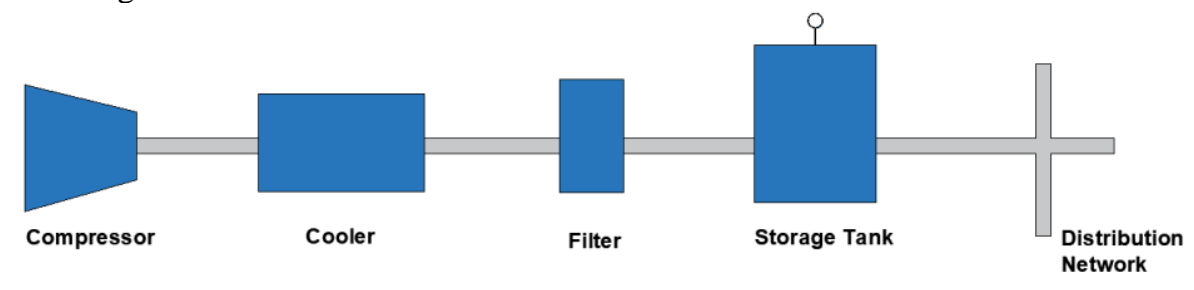

Fig. 1. CAS configuration considered

Mathematical formulations that describe performance of the sub-components were considered.

\subsection{Compressor}

According to [15], the power consumed by a compressor during normal operation can be modelled using (1).

$$
P_{\text {comp }}=\frac{P_{\text {air in }} \dot{V}_{\text {air }}}{\eta_{\text {Comp }}} \times \frac{n}{n-1} \times\left[\left(\frac{P_{\text {air out }}}{P_{\text {air in }}}\right)^{\frac{n-1}{n}}-1\right\rfloor
$$

The term ' $\eta$ ' refers to the efficiency of the compressor; however, if other equipment is involved in the compression process (motor, gearbox, etc.), their efficiency should also be taken into account.

Air was assumed to behave like an ideal gas, and the volumetric flow (capacity) was obtained from mass flow in at ambient conditions using ideal gas law shown in (2):

$$
\dot{V}_{\text {air }}=\frac{\dot{m}_{\text {air in }} \times R \times T_{\text {air in }}}{P_{\text {air in }}}
$$

It can be seen from the above equations that air inlet conditions, flow rate and compressor specifications (efficiency), are the main variables influencing the compressor performance.

\subsection{Cooler}

The mechanical compression of air causes an increase in its temperature. To reduce air temperature, coolers are typically installed after the final stage of compression. As air temperature decreases, water vapor in the air is condensed, separated, collected and drained from the system [14]. Anglani et al stated in [15] that the heat content of compressed air can be estimated using (3).

$$
Q_{C A}=\dot{m}_{C A} \times C_{p} \times\left(T_{C A}-T_{\text {air in }}\right)+\dot{m}_{v} h_{v}
$$

The temperature of compressed air leaving the compressor depends on air inlet temperature and on compression ratio. Temperature of air leaving the compressor was estimated using (4).

$$
T_{C A}=T_{\text {air in }} \times\left[\left(\frac{P \text { air out }}{P \text { air } \text { in }}\right)^{\frac{n-1}{n}}\right]
$$

Water vapor mass flow rate was estimated using (5).

$$
\dot{m}_{v}=U_{r} \rho_{\text {sin }}-\rho_{\text {soc }} \frac{T_{C A}}{T_{\text {air in }}} \times \frac{P_{\text {air in }}}{P_{\text {air out }}} \times \frac{1-U_{r} \frac{\rho_{\text {sin }}}{P_{\text {air in }}}}{1-\frac{\rho_{\text {soc }}}{P_{\text {air out }}}}
$$


An important parameter to calculate is the compressed air temperature at the cooler outlet. Usually, the cooler's effectiveness, which is a ratio that measures the cooler ability to transfer heat [16], is provided by the manufacturer. The effectiveness was obtained with (6).

$$
\varepsilon_{\text {cooler }}=\frac{T_{h, i}-T_{h, o}}{T_{h, i}-T_{c, i}}
$$

In equation (6) is valid when the mass flow rate of cooling fluid is larger than the mass flow rate of compressed air.

\section{$4.3 \quad$ Filters}

Compressed air usually contains particles, dust and oil (except for oil free-compressors). Removing these impurities is essential to provide improved compressed air quality, and to reduce component wear. For this purpose, filters are fitted in a CAS.

The air flow through the filter could cause a pressure drop [15]. This pressure drop is usually specified by the filter manufacturer. Over time, the efficiency of the filter might deteriorate and the pressure drop might increase. The pressure of air exiting the compressor was estimated using (7).

$$
P_{f, \text { out }}=P_{f, \text { in }}-P_{f, \text { drop }}
$$

Equation (7) indicates that the main variable describing filter performance is the pressure drop associated with it.

\subsection{Storage Tank}

Kleiser et al developed a dynamic model for the pressure and mass variation in the storage tank [17]. Variation of air mass inside was obtained using the conservation of mass principle, shown in (8).

$$
\frac{d m}{d t}=\dot{m}_{\text {Tank in }}-\dot{m}_{\text {Tank out }}
$$

The mass of air at any point in time was estimated by integrating equation (8) with respect to time. The result of this integration is shown in (9).

$$
m(t)=\int_{0}^{t}\left(\dot{m}_{\text {Tank in }}-\dot{m}_{\text {Tank out }}\right)+m_{\text {Tank initial }}
$$

The instantaneous pressure of air inside the tank was then estimated using the ideal gas law equation in (10):

\subsection{Pressure Drop in Pipes}

$$
P(t)=\frac{m(t) \times R \times T_{\text {tank }}}{V_{\text {tank }}}
$$

Anglani et al [15] estimated the pressure drop in a circular pipe with compressed air flowing through it using Darcy's formula shown in (11).

$$
\Delta P=f \frac{\rho_{\text {air }} \times L \times V_{\text {air }}^{2}}{2 \times D}
$$

$\mathrm{L}$ is the equivalent length of the pipe, which includes the actual length of the pipe and equivalent length of curved parts, valves, etc. The coefficient ' $\mathrm{f}$ ' represents the pipe friction factor, which will depend on flow characteristics. For a laminar flow (Reynolds number (Re) less than 4000) friction factor was estimated using (12).

$$
f=\frac{R e}{64}
$$

For the case of turbulent flow (which is most common in CAS), the friction factor was estimated with the Colebrook-White empiric formula, which is shown in equation (13). In this equation, $\varepsilon$ corresponds to the pipe absolute roughness, which depends on the material it is made of.

$$
\frac{1}{\sqrt{f}}=-2 \times \log \times\left[\frac{\frac{\varepsilon}{D}}{3.7}+\frac{2.51}{\operatorname{Re} \sqrt{f}}\right]
$$

\subsection{Combining the models}

The individual models described in sections 4.1 to 4.5 were implemented in MATLAB-Simulink. To properly describe the overall performance, these models are being combined to create a single integrated CAS model.

The next stage of the research will investigate the development of an integrated CAS model and a possible coupling with a model for heating, ventilation and air conditioning system.

\section{Discussion}

The authors aim to address all of the six identified research gaps. In response to the first gap mentioned in section 3 , a methodology is being created to support making decisions regarding suitable measures to save energy in CAS that may result in a useful and innovative tool for improved energy efficiency.

The second gap stated that most models in the literature focused on modelling either the supply or demand side of compressed air. The research described in this paper has so far investigated modelling individual CAS components. Future 
research may investigate integrating other plant energy usage (HVAC, lighting, etc.) with CAS, to obtain a model that describes a plant's total energy consumption.

The third gap, emphasized the need for smart energy management technologies to assist in automatic malfunction detection. This research will investigate machine learning techniques for optimizing the performance of CAS. An energy management system that detects abnormalities in energy performance, associates the abnormalities with suitable causes and sets up a troubleshooting procedure will be investigated.

Previous research regarding smart energy management and machine learning technologies for CAS, was not validated experimentally. Currently, an experimental set-up is being developed at the University of Portsmouth. The aim is therefore to investigate control, sensors, actuators and data processing tools to enable exchanging information and subsequently optimizing performance. This research will investigate validating intelligent energy management tools experimentally. This might help address the gaps relevant to CPCAS.

Last but not least, leak detection and treatment is an essential step to reduce waste in CAS. Technologies that are currently being used face several challenges such as: inability to operate during production, inaccuracy in sensors and noise coming from operating environments. Research into this area will investigate the creation of new intelligent leak detection techniques.

The modelling completed so far has provided an understanding of the equations and variables describing CAS components and processes. The main variables describing the performance of CAS will be investigated to discover their effects. This will provide an understanding of data required to describe the current state of CAS and that data can be used in the creation of new algorithms capable of detecting energy performance abnormalities. The algorithms will also associate each abnormality with a possible causes and will suggest the most probable cause. Such systems may also automate detection of faults and energy efficiency measures, which may be an innovative tool in CAS performance management. In that way, innovative intelligent methods will be created that reduce energy consumption in CAS.

In the future, research at the University of Portsmouth will combine ambient sensing information with AI and KM in real time. This should increase the efficiency of energy intensive manufacture. The work in this paper is a first step towards creating the AI systems to make automated decisions. KM system and will help to provide human operators with advice on how to maintain productivity and reduce energy usage. AI will make sense of that data and then will automatically act, for example to increase pressure or shut down a compressor if a leak is detected.

\section{Conclusions}

The initial stages of the research has consisted of identifying research gaps, defining problem characteristics and studying compressed air and buildings energy consumption. A preliminary literature review was completed. Mathematical models for CAS components were created and implemented in MATLAB. That is all the first step in creating innovative intelligent methods to reduce energy consumption in CAS. So as to increase the efficiency of energy intensive manufacture. The future work aims to use AI and ambient sensing to evaluate and monitor performance, use KM to decrease energy use and then to construct a collaborative interface for the human operators.

The models provided an understanding of critical variables that influence CAS performance. This is a first step towards determining the type of data required to build AI algorithms to reduce energy use and maintain productivity. AI will then be combined with KM, which will advise human operators on any actions required to. Some decisions might be made automatically, for example for safety reasons or to increase pressure or shut down a compressor if a leak is detected. Next steps will include collecting data from real CAS. This data will be analysed and the possibility of using pattern recognition to improve energy efficiency will be evaluated.

In addition to that, CAS modelling will be further investigated and the possibility of combining CAS with an HVAC model will be evaluated. The approach might enable the creation of new tools and methods to save energy in industrial facilities.

Future work will need to investigate AI [18-25] and Decision Making. The decision making research will include AHP and Preference Ranking Organization METHod for Enrichment of Evaluation (PROMETHEE) [26-34] and uncertainty will probably be denoted using probability functions, fuzzy set theory and percentages.

\section{Nomenclature}

$\begin{array}{ll}\mathbf{P}_{\text {comp }} & \text { Compressor input power } \\ \mathbf{P}_{\text {air in }} & \text { Absolute air pressure at compressor inlet } \\ \mathbf{V d o t}_{\text {air }} & \text { Volumetric flow rate of air } \\ \mathbf{\eta} & \text { Compressor efficiency (including drive system) } \\ \mathbf{P}_{\text {air out }} & \text { Absolute air pressure at compressor outlet } \\ \mathbf{n} & \text { Polytropic coefficient } \\ \dot{\mathbf{m}}_{\text {air in }} & \text { Air mass flow at compressor inlet } \\ \mathbf{T}_{\text {air in }} & \text { Temperature of Air at compressor inlet } \\ \mathbf{R} & \text { Gas Constant } \\ \mathbf{Q}_{\mathbf{C A}} & \text { Heat content of compressed air } \\ \mathbf{C}_{\mathbf{p}} & \text { Air specific heat } \\ \mathbf{T}_{\mathbf{C A}} & \text { Temperature of compressed air at compressor outlet } \\ \dot{\mathbf{m}}_{\mathbf{v}} & \text { Vapor water mass flow rate } \\ \mathbf{h}_{\mathbf{v}} & \text { Latent heat of condensation for water vapor } \\ \mathbf{U}_{\mathbf{r}} & \text { Relative Humidity } \\ \boldsymbol{\rho}_{\text {s, in }} & \text { Water vapor content in saturation at compressor inlet conditions } \\ \boldsymbol{\rho}_{\mathbf{s}, \text { out }} & \text { Water vapor content in saturation at compressor outlet conditions }\end{array}$




$\begin{array}{ll}\boldsymbol{\varepsilon} \text { cooler } & \text { Cooler heat transfer effectiveness } \\ \mathbf{T}_{\mathbf{h}, \mathbf{i}} & \text { Temperature of hot fluid entering the cooler } \\ \mathbf{T}_{\mathbf{h}, \mathbf{o}} & \text { Temperature of hot fluid exiting the cooler } \\ \mathbf{T}_{\mathbf{c}, \mathbf{i}} & \text { Temperature of cold fluid entering the cooler } \\ \mathbf{P}_{\mathbf{f}, \text { out }} & \text { Pressure of Air at filter outlet } \\ \mathbf{P}_{\mathbf{f}, \mathbf{i n}} & \text { Pressure of Air at filter inlet } \\ \mathbf{P}_{\text {drop }} & \text { Pressure drop in filter } \\ \dot{\mathbf{m}}_{\text {tank in }} & \text { Air mass flow rate at storage tank inlet } \\ \dot{\mathbf{m}}_{\text {tank out }} & \text { Air mass flow rate at storage tank outlet } \\ \mathbf{m}_{\text {tank intial }} & \text { Initial mass of air in storage tank } \\ \mathbf{T}_{\text {tank }} & \text { Temperature of Air in storage tank } \\ \mathbf{V}_{\text {tank }} & \text { Volume of storage tank } \\ \mathbf{f} & \text { Piper friction factor } \\ \boldsymbol{\rho}_{\text {air }} & \text { Air density } \\ \mathbf{L} & \text { Pipe length } \\ \mathbf{D} & \text { Pipe Diameter } \\ \mathbf{V}_{\text {air }} & \text { Air velocity in pipe } \\ \mathbf{R e} & \text { Reynolds Number } \\ \boldsymbol{\varepsilon} & \text { Pipe Roughness } \\ \end{array}$

\section{Acknowledgment}

Research in this paper was funded by the DTA3/COFUND Marie Skłodowska-Curie PhD Fellowship programme partly funded by the Horizon 2020 European Programme.

\section{References}

1. Sanders DA, Robinson DC, Hassan M, Haddad M, Gegov A, Ahmed N. Making decisions about saving energy in compressed air systems using ambient intelligence and artificial intelligence. Adv Intell Syst Comput. 2018;869(September): 1229-36.

2. Thabet M, Sanders D, Beccera V, Tewkesbury G, Haddad M, Barker T. (in press) Intelligent Energy Management of Compressed Air Systems. IEEE Proceedings of $10^{\text {th }}$ International Conference on Intelligent Systems. Varna, Bulgaria 2020

3. Saidur R, Rahim NA, Hasanuzzaman M. A review on compressed-air energy use and energy savings. Renew Sustain Energy Rev [Internet]. 2010;14(4):1135-53.

4. Fridén H, Bergfors L, Björk A, Mazharsolook E. Energy and LCC optimised design of compressed air systems: A mixed integer optimisation approach with general applicability. Proc - 2012 14th Int Conf Model Simulation, UKSim 2012. 2012;(Lcc):491-6.

5. Murphy S, Kissock K. Simulating Energy Efficient Control of Multiple-Compressor Compressed Air Systems. Proc Ind Energy Technol Conf. 2015;

6. Benedetti M, Cesarotti V, Introna V, Serranti J. Energy consumption control automation using Artificial Neural Networks and adaptive algorithms: Proposal of a new methodology and case study. Appl Energy [Internet]. 2016;165:60-71.

7. Bonfá F, Benedetti M, Ubertini S, Introna V, Santolamazza A. New efficiency opportunities arising from intelligent real time control tools applications: the case of Compressed Air Systems' energy efficiency in production and use. Energy Procedia [Internet]. 2019;158:4198-203.

8. Santolamazza A, Cesarotti V, Introna V. Anomaly detection in energy consumption for Condition-Based maintenance of Compressed Air Generation systems: an approach based on artificial neural networks. IFAC-PapersOnLine [Internet]. 2018;51(11):1131-6.

9. Santolamazza A, Cesarotti V, Introna V. Evaluation of Machine Learning techniques to enact energy consumption control of Compressed Air Generation in production plants. Proc Summer Sch Fr Turco. 2018;(2004):79-86.

10. Boehm R, Franke J. Demand-side-management by Flexible Generation of Compressed Air. Procedia CIRP [Internet]. 2017;63:195-200. 11. Ghorbanian K, Gholamrezaei M. An artificial neural network approach to compressor performance prediction. Appl Energy [Internet]. 2009;86(7-
8):1210-21.

12. Nehler T, Parra R, Thollander P. Implementation of energy efficiency measures in compressed air systems: barriers, drivers and non-energy benefits. Energy Effic. 2018;11(5):1281-302.

13. Dudić S, Ignjatović I, Šešlija D, Blagojević V, Stojiljković M. "Leakage quantification of compressed air using ultrasound and infrared thermography," Meas. J. Int. Meas. Confed., vol. 45, no. 7, pp. 1689-1694, 2012.

14. Lawrence Berkeley. Compressed Air: a sourcebook for industry. 2003;1-128.

15. Anglani N, Bossi M, Quartarone G. Energy conversion systems: The case study of compressed air, an introduction to a new simulation toolbox. 2012 IEEE Int Energy Conf Exhib ENERGYCON 2012. 2012;32-8.

16. Bergman T, Lavine A, Incropera F, Dewitt D. Fundamentals of Heat and MAss Transfer. John Wiley \& Sons; 2011.1076 p.

17. Kleiser G, Rauth V. Dynamic Modelling of Compressed Air Energy Storage for Small-Scale Industry Applications. Int J Energy Eng. 2013;3(3):12737 .

18. Sanders D, Gegov A, Ndzi D. Knowledge-based expert system using a set of rules to assist a tele-operated mobile robot, in Studies in Computational Intelligence, (eds) Y. Bi, S. Kapoor, and R. Bhatia, 2018, 751, Springer, 2018b, pp. 371-392.

19. Sanders D, Sanders H, Gegov A, Ndzi D. Rule-based system to assist a tele-operator with driving a mobile robot. Lecture Notes in Networks and Systems, 16, Springer, pp. 599-615, 2018.

20. Sanders D, Okonor OM, Langner M, Hassan Sayed M, Khaustov SA, Omoarebun P. Using a simple expert system to assist a powered wheelchair user. in Y Bi, R Bhatia \& S Kapoor (eds), Advances in Intelligent Systems and Computing, vol. 1037, Springer, pp. 662-379.

21. Gegov, A., Gobalakrishnan, N.and Sanders, D. A.: Rule base compression in fuzzy systems by filtration of non-monotonic rules, Journal of Intelligent \& Fuzzy Systems, 27(4), 2029-2043, (2014).

22. Sanders D, Gegov A, Haddad M, Ikwan F, Wiltshire D, Tan YC. A Rule-based Expert System to decide on direction and speed of a powered wheelchair. IEEE Proceedings of the SAI Conference on IntelliSys. London, U.K., 2018a, pp. 426- 433.

23. Sanders D, Gegov A, Haddad M, Ikwan F, Wiltshire D, Tan YC. A rule-based expert system to decide on direction and speed of a powered wheelchair. in K Arai, S Kapoor \& R Bhatia (eds), Advances in Intelligent Systems and Computing, vol. 868, Springer, pp. $822-838$.

24. Sanders D. Recognizing shipbuilding parts using artificial neural networks and Fourier descriptors, Proceedings of the Institution of Mechanical Engineers - Part B- Journal of Engineering Manufacture, 223(3), 337-342, (2009)a.

25. Sanders D. Using self-reliance factors to decide how to share control between human powered wheelchair drivers and ultrasonic sensors, IEEE Transactions on Neural Systems and Rehabilitation Engineering, 25(8), 1221-1229, (2017).

26. Haddad M, Sanders D, Bausch N, TewkesburyvvG, Gegov A, Hassan Sayed M. Learning to make intelligent decisions using an Expert System for the intelligent selection of either PROMETHEE II or the Analytical Hierarchy Process. in K Arai, S Kapoor \& R Bhatia (eds), Advances in Intelligent Systems and Computing, vol. 868, Springer, pp. 1303-1316.

27. Haddad MJM, Sanders D, Gegov A, Hassan Sayed M, Huang Y, Al-Mosawi M. Combining multiple criteria decision making with vector manipulation to decide on the direction for a powered wheelchair. in Y Bi, R Bhatia \& S Kapoor (eds), Advances in Intelligent Systems and Computing, vol. 1037, Springer, pp. 680-693, . 
28. Haddad MJM, Sanders D, Tewkesbury G, Gegov A, Hassan Sayed M, Ikwan F. Initial results from using Preference Ranking Organization METHods for Enrichment of Evaluations to help steer a powered wheelchair. in Y Bi, R Bhatia \& S Kapoor (eds), Advances in Intelligent Systems and Computing, vol. 1037, Springer, pp. 648-661.

29. Sanders D, Robinson DC, Hassan Sayed M, Haddad MJM, Gegov A, Ahmed N. Making decisions about saving energy in compressed air systems using Ambient Intelligence and Artificial Intelligence. in K Arai, S Kapoor \& R Bhatia (eds), Advances in Intelligent Systems and Computing, vol.

30. Haddad M, Sanders D, Bausch N. Selecting a Robust Decision Making Method to Evaluate Employee Performance, International Journal of Management and Decision Making. 8(4),333 -351, (2019).

31. Haddad M, Sanders D. The Behavior of Three Discrete Multiple Criteria Decision Making Methods in the Presence of Uncertainty, Operations Research Perspectives, to be published.

32. Haddad MJM, Sanders D, Bausch N. Selecting a robust decision making method to evaluate employee performance, International Journal of Management and Decision Making, vol. 18, no. 4, pp. 333-351.

33. Haddad MJM, Sanders D. Selecting a best compromise direction for a powered wheelchair using PROMETHEE, IEEE Transactions on Neural Systems and Rehabilitation Engineering, vol. 27, no. 2, pp. 228-235. https://doi.org/10.1109/TNSRE.2019.2892587

34. Sanders D, Robinson DC, Hassan Sayed M, Haddad MJM, Gegov A, Ahmed N. Making decisions about saving energy in compressed air systems using Ambient Intelligence and Artificial Intelligence. in K Arai, S Kapoor \& R Bhatia (eds), Advances in Intelligent Systems and Computing, vol. 869 , Springer, pp. 1229-1236. 